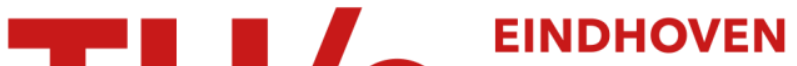 UNIVERSITY OF TECHNOLOGY
}

\section{Transient behaviour and stability points of the Poiseuille flow of a KBKZ-fluid}

Citation for published version (APA):

Aarts, A. C. T., \& Ven, van de, A. A. F. (1994). Transient behaviour and stability points of the Poiseuille flow of a KBKZ-fluid. (RANA : reports on applied and numerical analysis; Vol. 9410). Eindhoven University of Technology.

Document status and date:

Published: 01/01/1994

\section{Document Version:}

Publisher's PDF, also known as Version of Record (includes final page, issue and volume numbers)

\section{Please check the document version of this publication:}

- A submitted manuscript is the version of the article upon submission and before peer-review. There can be important differences between the submitted version and the official published version of record. People interested in the research are advised to contact the author for the final version of the publication, or visit the $\mathrm{DOI}$ to the publisher's website.

- The final author version and the galley proof are versions of the publication after peer review.

- The final published version features the final layout of the paper including the volume, issue and page numbers.

Link to publication

\section{General rights}

Copyright and moral rights for the publications made accessible in the public portal are retained by the authors and/or other copyright owners and it is a condition of accessing publications that users recognise and abide by the legal requirements associated with these rights.

- Users may download and print one copy of any publication from the public portal for the purpose of private study or research.

- You may not further distribute the material or use it for any profit-making activity or commercial gain

- You may freely distribute the URL identifying the publication in the public portal.

If the publication is distributed under the terms of Article 25fa of the Dutch Copyright Act, indicated by the "Taverne" license above, please follow below link for the End User Agreement:

www.tue.nl/taverne

Take down policy

If you believe that this document breaches copyright please contact us at:

openaccess@tue.nl

providing details and we will investigate your claim. 


\section{EINDHOVEN UNIVERSITY OF TECHNOLOGY}

Department of Mathematics and Computing Science

RANA 94-10

June 1994

Transient behaviour and stability points of the Poiseuille flow of a KBKZ-fluid

by

A.C.T. Aarts and A.A.F. van de Ven 
Reports on Applied and Numerical Analysis

Department of Mathematics and Computing Science Eindhoven University of Technology

P.O. Box 513

5600 MB Eindlhoven

The Netherlands

ISSN: 0926-4507 


\title{
Transient behaviour and stability points of the Poiseuille flow of a KBKZ-fluid
}

\author{
A.C.T. Aarts and A.A.F. van de Ven \\ Eindhoven University of Technology \\ Department of Mathematics and Computing Science \\ P.O. Box 513, 5600 MB Eindhoven, The Netherlands
}

\begin{abstract}
The Poiseuille flow of a KBKZ-fluid, being a nonlinear viscoelastic model for a polymeric fluid, is studied. The flow starts from rest and especially the transient phase of the flow is considered. It is shown that under certain conditions the steady flow equation has three different equilibrium points. The stability of these points is investigated. It is proved that two points are stable, whereas the remaining one is unstable, leading to several peculiar phenomena such as discontinuities in the velocity gradient near the wall of the pipe ('spurt') and hysteresis. Our theoretical results are confirmed by numerical calculations of the velocity gradient.
\end{abstract}

\section{Introduction}

In two papers [1] and [2], Malkus et al. analyzed a striking novel phenomenon in shearing flows of non-Newtonian fluids called the 'spurt' phenomenon. They associated spurt with a material property of the polymeric fluid in contrast to the widely accepted explanation of spurt being the failure of the fluid to adhere to the wall ('wall slip'). In their analysis, this effect is due to a jump in the steady strain rate profile, leading to a dramatic increase in the volumetric flow rate. This phenomenon was also observed experimentally by Vinogradov et al. [3] in the flow of polymeric fluids through tubes. Similar effects are known in industrial applications, such as injection moulding or extrusion of polymeric melts through a capillary to produce fibres. The occurrence of a 'spurt jump' causes surface distortion of the extrudate, making the final industrial product more or less worthless. Therefore, the determination of a critical pressure gradient or volumetric flow rate, below which no spurt occurs is of great practical value in the manufacturing of polymeric melts in chemical industry.

Malkus et al. used a non-Newtonian fluid model (the so-called Johnson-SegelmanOldroyd fluid) that was described by (nonlinear) differential equations. Moreover, they 
restricted themselves to a two-dimensional shear flow trough a slit die. Although the results of Malkus et al. did explain (amongst other things) the spurt phenomenon quite satisfactorily, we are of the opinion that a polymeric fluid is more adequately described by a nonlinear viscoelastic model than by a fluid model. Such a viscoelastic model will lead to integrodifferential equations (due to the hereditary effect) rather than differential equations. To describe the elastic response of the dissolved polymer, we have exploited a so-called Kaye-Bernstein-Kearsly-Zapas (KBKZ)-model (see [4, p. 141]) with an extra viscous term, due to the small-molecule solvent. As a generalisation of [2] we consider the three-dimensional axisymmetric shear flow through a pipe (Poiseuille flow). Since in simple shearing the second normal stress difference may be neglected, we use Wagner's modification (see [4, p. 209]) of the KBKZ-model. The constitutive equation contains a hereditary integral, the kernel of which is taken from the form as explored by Papanastasiou (see [4, p. 213]). The extra viscous term will dominate the initial response of the fluid. As we shall show in the sequel, the occurrence of this term is essential for our further considerations.

The description of the Poiseuille flow of such a fluid leads to an integrodifferential equation for the total amount of shear for a given pressure gradient. This equation will be derived in Section 2. It is assumed that the pressure gradient is either constant from the start or reaches a stationary value within a restricted time interval. If the pressure gradient is prescribed, the volumetric flow rate is still an unknown of the problem for which a global relation will be derived. The flow starts at $t=0$ ( for $t<0$ the fluid is at rest) by a sudden application of the pressure gradient. After the transient phase in which the flow is not stationary, the flow reaches a steady state profile. Due to the high viscosity of the fluid, inertia terms will be neglected throughout.

In Section 3 the steady state solution is derived, that is, we have calculated the stationary velocity gradient $\omega$ as function of the radial coordinate $r(\omega=\omega(r))$. In addition, this gradient also depends upon the stationary value $\bar{f}$ of the pressure gradient. However, this solution is not always unique, depending on the value of the quantity $F=F(r):=r \bar{f} / 2$, which represents the magnitude of the shear stress at $r$. For a certain range of values for $F$, three distinct solutions for the steady state velocity gradient exist. The first essential question is which of these three states will be attained by the fluid after the transient phase. The second question concerns the (in)stability of these states (of course this question is related to the first one). The answer to this stability question will be given in Section 4 . In Section 5 we present some results of numerical simulations. These numerical computations confirm the stability of the different states and show which particular steady state will be attained in case there are two stable ones. Moreover, the influence of the hereditary effect is observed by changing the stationary value of the pressure gradient. This involves understanding phenomena related to spurt, such as shape memory and hysteresis. Finally, in Section 6 we recapitulate the main conclusions of our paper. 


\section{Mathematical formulation of the Poiseuille flow of a KBKZ-fluid}

The flow of an incompressible viscoelastic fluid under isothermal conditions is governed by the conservation of mass

$$
\nabla \cdot \mathrm{v}=0
$$

and the balance of linear momentum

$$
\nabla \cdot \mathcal{T}+\rho \mathbf{b}=\rho\left(\frac{\partial \mathbf{v}}{\partial t}+(\mathbf{v} \cdot \nabla) \mathbf{v}\right)
$$

Here, $\rho$ is the (constant) fluid density, $\mathbf{b}$ the body force per unit of mass, $\mathbf{v}$ the particle velocity and $\mathcal{T}$ the total (symmetric) stress tensor. Later on we shall show that for strongly viscous fluids the inertia forces, represented by the right-hand side of $(2.2)$, can be neglected.

The characteristic response of the material is described by the constitutive equation for the stress. For viscoelastic fluids with fading memory, the stress depends on the deformation history. If a polymer solution contains a small-molecule solvent, this solvent will generally respond in a viscous manner to any signal, separately from the elastic response due to the dissolved polymer. Therefore, it is assumed that the extra stress in the fluid consists of a viscous component and an isotropic elastic one, namely

$$
\mathcal{T}+p \mathcal{I}=2 \eta_{s} \mathcal{D}+\mathcal{S}_{p}
$$

Here, $p$ is the pressure, $\mathcal{I}$ the unit tensor and $\mathcal{D}$ is the rate-of-deformation tensor defined by

$$
\mathcal{D}=\frac{1}{2}\left(\nabla \mathbf{v}+(\nabla \mathbf{v})^{T}\right)
$$

Moreover, $\eta_{s}$ is the coefficient of Newtonian viscosity. Finally, the elastic part $\mathcal{S}_{p}$ characterizes the polymer contribution, and this part is here assumed to be described by a KBKZ-model (cf. [4, p. 141]), which in its general form reads

$$
\mathcal{S}_{p}=\int_{-\infty}^{t}\left(\frac{\partial U}{\partial \mathrm{I}_{\mathcal{C}^{-1}}} \mathcal{C}^{-1}-\frac{\partial U}{\partial \mathrm{I}_{\mathcal{C}}} \mathcal{C}\right) d \tau
$$

Here, $\mathcal{C}$ is the strain tensor (see definition (2.7)), $\mathcal{C}^{-1}$ (the Finger tensor) is its inverse, $\mathrm{I}_{\mathcal{C}}$ and $\mathrm{I}_{\mathcal{C}^{-1}}$ are the first invariants of these tensors, and the potential $U$ is in general a scalar function of $\mathrm{I}_{\mathcal{C}}, \mathrm{I}_{\mathcal{C}^{-1}}$ and $t-\tau$. We use Wagner's modification of the KBKZ model for shearing flows by choosing $\partial U / \partial \mathrm{I}_{\mathcal{C}^{-1}}=m(t-\tau) K\left(\mathrm{I}_{\mathcal{C}^{-1}}\right)$ and $\partial U / \partial \mathrm{I}_{\mathcal{C}}=0$ (see $[4$, p. 209]). If we use the kernel $K$ explored by Papanastasiou et al. (see [4, p. 213]) and restrict ourselves to one relaxation rate $\lambda$, then equation $(2.5)$ becomes

$$
\mathcal{S}_{p}=\int_{-\infty}^{t} \frac{\mu \lambda}{c+\mathrm{I}_{\mathcal{C}^{-1}}} \mathcal{C}^{-1} e^{-\lambda(t-\tau)} d \tau
$$


where $c, \mu$ and $\lambda$ are material constants. When a material particle moves from position $\tilde{\mathbf{x}}$ at time $\tau$ to $\mathbf{x}$ at time $t(\tau \leq t)$, the strain tensor $\mathcal{C}$ is given by

$$
\mathcal{C}=\mathcal{F}^{\mathrm{T}} \mathcal{F}, \quad \mathcal{F}=\frac{\partial \tilde{\mathbf{x}}}{\partial \mathbf{x}}
$$

and its inverse is

$$
\mathcal{C}^{-1}=\mathcal{F}^{-1} \mathcal{F}^{-\mathrm{T}}
$$

In this paper we study an axisymmetric shear flow in a tube with radius $R$, starting at time $t=0$. With this flow aligned along the $z$-axis the flow parameters are independent of the axial coordinate $z$ and the azimuthal coordinate $\theta$. Hence, the velocity takes the form

$$
\mathbf{v}=v(r, t) H(t) \mathbf{e}_{z},
$$

where $H$ is the (Heaviside) step function. The conservation of mass is now automatically satisfied. The no-slip boundary condition at the wall and regularity of the velocity at the axis require

$$
v(R, t)=0
$$

and

$$
\frac{\partial v}{\partial r}(0, t)=0
$$

respectively. To determine the stress components we need the strain tensor $\mathcal{C}$ and its inverse. With the momentary position at time $t$ represented in cylindrical coordinates by $\mathbf{x}=r \mathbf{e}_{r}+z \mathbf{e}_{z}$, the position of the same particle at an earlier time $\tau$ is

$$
\tilde{\mathbf{x}}=r \mathbf{e}_{r}+\left(z-\int_{\tau}^{t} v(r, s) H(s) d s\right) \mathbf{e}_{z} .
$$

Then (2.8) yields

$$
\mathcal{C}^{-1}=\left(\begin{array}{ccc}
1 & 0 & -\gamma \\
0 & 1 & 0 \\
-\gamma & 0 & 1+\gamma^{2}
\end{array}\right)
$$

and $I_{\mathcal{C}^{-1}}=3+\gamma^{2}$, where

$$
\gamma=\gamma(r, t, \tau)=-\int_{\tau}^{t} \frac{\partial v}{\partial r}(r, s) H(s) d s, \quad t \geq \tau,
$$

is the magnitude of the shear strain at time $t$ when the strain is applied at time $\tau$. For $\tau<0$ no motion is observed, hence

$$
\gamma(r, t, \tau)=\gamma(r, t, 0), \quad \tau<0 .
$$


In terms of $\gamma$, the stress components according to (2.3) and (2.6) become

$$
\begin{aligned}
& T_{r r}=T_{\theta \theta}=-p+\mu \lambda \int_{-\infty}^{t} \frac{1}{c+3+\gamma^{2}(r, t, \tau)} e^{-\lambda(t-\tau)} d \tau \\
& T_{z z}=-p+\mu \lambda \int_{-\infty}^{t} \frac{1+\gamma^{2}(r, t, \tau)}{c+3+\gamma^{2}(r, t, \tau)} e^{-\lambda(t-\tau)} d \tau \\
& T_{r z}=\eta_{s} \frac{\partial v}{\partial r}-\mu \lambda \int_{-\infty}^{t} \frac{\gamma(r, t, \tau)}{c+3+\gamma^{2}(r, t, \tau)} e^{-\lambda(t-\tau)} d \tau \\
& T_{r \theta}=T_{\theta z}=0
\end{aligned}
$$

where $p=p(r, z, t)$. The balance of linear momentum (2.2), with $\rho \mathrm{b}=0$, is satisfied if the pressure $p$ takes the form

$$
p(r, z, t)=-f(t) z+p_{0}(r, t)
$$

with

$$
p_{0}(r, t)=\mu \lambda \int_{-\infty}^{t} \frac{1}{c+3+\gamma^{2}(r, t, \tau)} e^{-\lambda(t-\tau)} d \tau+P_{0}(t)
$$

and if the shear stress $T_{r z}$ equals

$$
T_{r z}=-\frac{1}{2} r f(t)+\frac{\rho}{r} \int_{0}^{r} \xi \frac{\partial v}{\partial t}(\xi, t) d \xi
$$

Here, $f$ is the pressure gradient driving the flow and $P_{0}$ is a further irrelevant pressure term. Substitution of (2.19) into (2.16) results in the following relation between the velocity and the pressure gradient:

$$
\begin{aligned}
\eta_{s} \frac{\partial v}{\partial r}(r, t)-\mu \frac{\gamma(r, t, 0)}{c+3+\gamma^{2}(r, t, 0)} e^{-\lambda t}-\mu \lambda \int_{0}^{t} \frac{\gamma(r, t, \tau)}{c+3+\gamma^{2}(r, t, \tau)} e^{-\lambda(t-\tau)} d \tau \\
=-\frac{1}{2} r f(t)+\frac{\rho}{r} \int_{0}^{r} \xi \frac{\partial v}{\partial t}(\xi, t) d \xi, \quad 0 \leq r \leq R, \quad t>0 .
\end{aligned}
$$

Equation (2.20) can be made dimensionless by scaling length by $R$ and time by $\lambda^{-1}$. Furthermore we introduce dimensionless variables $\tilde{v}, \tilde{f}$ and $\tilde{\gamma}$ by writing $v=\lambda R \sqrt{c+3} \tilde{v}$, $\gamma=\sqrt{c+3} \tilde{\gamma}, f=\mu \tilde{f} /(R \sqrt{c+3})$, and the two dimensionless material parameters $\varepsilon$ and $\alpha$ by

$$
\varepsilon=(c+3) \frac{\eta_{s} \lambda}{\mu}, \quad \alpha=(c+3) \frac{\rho R^{2} \lambda^{2}}{\mu}
$$

Then (2.20) turns into its dimensionless form, reading (since no confusion will arise we omit the tilde)

$$
\begin{aligned}
-\varepsilon \frac{\partial v}{\partial r}(r, t)+h(\Gamma(r, t)) e^{-t}+\int_{0}^{t} h(\gamma(r, t, \tau)) e^{-(t-\tau)} d \tau & \\
= & \frac{1}{2} r f(t)-\frac{\alpha}{r} \int_{0}^{r} \xi \frac{\partial v}{\partial t}(\xi, t) d \xi, \quad 0 \leq r \leq 1, \quad t>0,
\end{aligned}
$$


where the function $h$ is defined by

$$
h(x)=\frac{x}{1+x^{2}},
$$

while the variable $\Gamma$ represents the total amount of shear

$$
\Gamma(r, t)=\gamma(r, t, 0)=-\int_{0}^{t} \frac{\partial v}{\partial r}(r, s) d s, \quad 0 \leq r \leq 1, \quad t \geq 0 .
$$

So $\gamma$ can be expressed in terms of $\Gamma$ as

$$
\gamma(r, t, \tau)=\Gamma(r, t)-\Gamma(r, \tau), \quad 0 \leq r \leq 1, \quad 0 \leq \tau \leq t .
$$

The parameter $\varepsilon$ represents a ratio of the Newtonian viscosity $\eta_{s}$ to the shear viscosity $\mu / \lambda$ and the quotient $\alpha / \varepsilon$ corresponds to the Reynolds number. For the highly elastic and viscous polymers we describe in this paper, $\alpha \ll 1$. Thus, the last term in the right-hand side of (2.22) may be neglected. In the original equation this amounts to the neglect of the inertia terms. With the last term neglected and after division by $r / 2$, equation $(2.22)$ becomes

$$
-\varepsilon \frac{2}{r} \frac{\partial v}{\partial r}(r, t)+\frac{2}{r} h(\Gamma(r, t)) e^{-t}+\frac{2}{r} \int_{0}^{t} h(\gamma(r, t, \tau)) e^{-(t-\tau)} d \tau=f(t) .
$$

The right-hand side of (2.26) is independent of $r$. Therefore, it is necessary that

$$
-\lim _{r \downarrow 0} \frac{1}{r} \frac{\partial v}{\partial r}(r, t)=: u(t)
$$

exists (as we shall assume here). By letting $r \downarrow 0$ in (2.26) and performing one integration by parts, we obtain an expression for the pressure gradient $f(t)$ in terms of the function $u(t)$ defined above, i.e.

$$
f(t)=2 \varepsilon u(t)+2 \int_{0}^{t} u(\tau) e^{-(t-\tau)} d \tau
$$

The inverse of this relation is

$$
u(t)=\frac{1}{2 \varepsilon} f(t)-\frac{1}{2 \varepsilon^{2}} \int_{0}^{t} f(\tau) \exp \left[-\frac{1+\varepsilon}{\varepsilon}(t-\tau)\right] d \tau,
$$

which can be used to calculate the function $u$ when $f$ is given. The boundary conditions pertinent to (2.26) read in dimensionless form

$$
v(1, t)=\frac{\partial v}{\partial r}(0, t)=0, \quad t>0
$$

We conclude this section by deriving an expression in terms of $\Gamma$ for the volumetric flux $Q$ defined by

$$
Q(t)=2 \pi \int_{0}^{R} v(r, t) r d r
$$


or in dimensionless form

$$
\tilde{Q}(t)=2 \int_{0}^{1} \tilde{v}(r, t) r d r
$$

where $\tilde{Q}=Q /\left(\pi R^{3} \lambda \sqrt{c+3}\right)$. After one integration by parts with the aid of $(2.30)$, relation (2.32) transforms into (omitting the tilde)

$$
Q(t)=-\int_{0}^{1} r^{2} \frac{\partial v}{\partial r}(r, t) d r
$$

Integration of (2.33) with respect to $t$ yields a relation between the total amount of shear and the volumetric flow, of the form

$$
\int_{0}^{t} Q(\tau) d \tau=\int_{0}^{1} r^{2} \Gamma(r, t) d r
$$

Since, according to (2.26), $\Gamma$ is determined by $f(t)$, relation (2.34) provides an (implicit) relation between $Q$ and $f$.

\section{The steady state solution}

In this section we investigate the asymptotic behaviour of the flow profile as $t \rightarrow \infty$. It turns out that the velocity profile reaches a steady state (in which the flow variables are independent of the time) as $t \rightarrow \infty$. This steady state plays an important role in the explanation of the spurt effect. The steady state velocity profile will be expressed in terms of the steady state velocity gradient $\omega$, defined by

$$
\omega(r)=\lim _{t \rightarrow \infty}-\frac{\partial v}{\partial r}(r, t), \quad \omega(0)=0 .
$$

From the stability analysis in Section 4 it follows that this steady state velocity gradient exists. In this section we shall derive an equation for $\omega$. Let the pressure gradient $f(t)$ be prescribed and take $\alpha=0$ in equation (2.22). For $t \rightarrow \infty$ this equation turns into a relation between the steady state velocity gradient $\omega$ and the given pressure gradient $f$.

Proposition 3.1 The steady state velocity gradient $\omega$ defined by (3.1) satisfies

$$
\varepsilon \omega(r)+\omega(r) \int_{0}^{\infty} \frac{\tau e^{-\tau}}{1+\omega^{2}(r) \tau^{2}} d \tau=\frac{1}{2} r \bar{f}, \quad 0 \leq r \leq 1
$$

if

$$
\bar{f}=\lim _{t \rightarrow \infty} f(t)
$$

exists. 
Proof: Given the existence of $\bar{f}$, suppose that also $\omega(r)$, as defined by (3.1), exists (this will be confirmed by the results of Section 4). Then for $t \rightarrow \infty$, the first and last terms of equation (2.22) (with $\alpha=0$ ) tend to $\varepsilon \omega(r)$ and $r \bar{f} / 2$. Since $h$ is bounded, the function $h(\Gamma(r, t)) e^{-t}$ vanishes for $t \rightarrow \infty$. Hence, to verify that equation (2.22) turns into equation (3.2) as $t \rightarrow \infty$, it remains to prove that

$$
\lim _{t \rightarrow \infty} \int_{0}^{t}[h(\gamma(r, t, \tau))-h(\omega(r)(t-\tau))] e^{-(t-\tau)} d \tau=0 .
$$

Let $\delta>0$, then there exists a time $T_{1}$ such that

$$
\left|\frac{\partial v}{\partial r}(r, t)+\omega(r)\right|<\delta, \quad t>T_{1} .
$$

Choose $t_{0}>T_{1}$ arbitrary and let $T=\max \left\{t_{0}, T_{2}\right\}$, where $T_{2}$ is such that

$$
e^{-t} \int_{0}^{t_{0}}|h(\gamma(r, t, \tau))-h(\omega(r)(t-\tau))| e^{\tau} d \tau<\delta, \quad t>T_{2}
$$

For all $t>t_{0}$ the remaining integral over $\left[t_{0}, t\right]$ is bounded by

$$
\int_{t_{0}}^{t}|h(\gamma)-h(\omega(t-\tau))| e^{-(t-\tau)} d \tau \leq \int_{t_{0}}^{t}|\gamma(r, t, \tau)-\omega(r)(t-\tau)| e^{-(t-\tau)} d \tau<\delta
$$

since (3.5) implies

$$
|\gamma(r, t, \tau)-\omega(r)(t-\tau)|<\delta(t-\tau), \quad t_{0} \leq \tau \leq t .
$$

The inequalities (3.6) and (3.7) imply that the integral in (3.4) is bounded by $2 \delta$, for all $t>T$. This completes the proof.

We introduce the integral $J$ defined by

$$
J(\omega)=\omega \int_{0}^{\infty} \frac{\tau e^{-\tau}}{1+\omega^{2} \tau^{2}} d \tau .
$$

Then the steady state velocity gradient can be determined for each $r \epsilon[0,1]$ by solving $\omega=\omega(r)$ from

$$
\varepsilon \omega+J(\omega)=F,
$$

where $F$ is given by $F(r)=r \bar{f} / 2$. The steady state velocity profile $\bar{v}(r)$ is next obtained by integration of $\omega(r)$ using the boundary condition at the wall. In the Appendix it is shown that the function

$$
\mathcal{F}(\omega ; \varepsilon):=\varepsilon \omega+J(\omega), \quad \omega \geq 0,
$$

is nonmonotonic in $\omega$ when $0 \leq \varepsilon<\varepsilon_{1}$, where $\varepsilon_{1}=-J^{\prime}\left(\omega^{* *}\right)=0.02886$. In Fig. 1 the function $\mathcal{F}(\omega ; \varepsilon)$ is plotted if $0<\varepsilon<\varepsilon_{1}$. Since the viscosity $\eta_{s}$ of the small-molecule solvent is small in comparison to the shear viscosity $\mu / \lambda$, we will henceforth assume that $0<\varepsilon<\varepsilon_{1}$. 


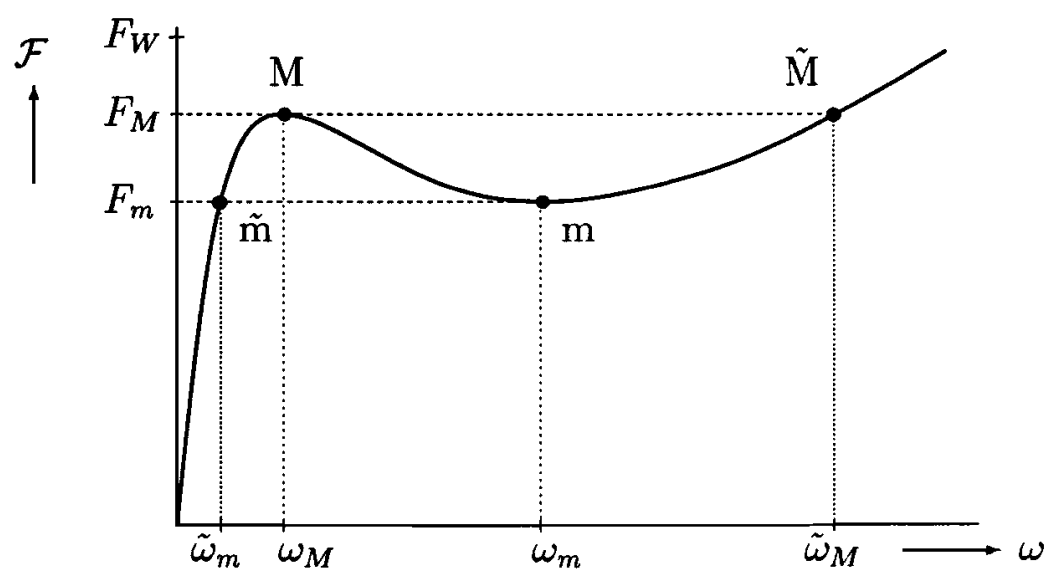

Figure 1 The function $\mathcal{F}(\omega ; \varepsilon)=\varepsilon \omega+J(\omega)$, if $0<\varepsilon<\varepsilon_{1}$. In steady flow the velocity gradient $\omega$ satisfies $\mathcal{F}(\omega ; \varepsilon)=F$, where $F$ denotes the shear stress.

Then the function $\mathcal{F}(\omega ; \varepsilon)$ has two extreme values, a maximum $F_{M}=\varepsilon \omega_{M}+J\left(\omega_{M}\right)$ at $\omega=\omega_{M}$ and a minimum $F_{m}=\varepsilon \omega_{m}+J\left(\omega_{m}\right)$ at $\omega=\omega_{m}$; see Fig. 1. If $\varepsilon \uparrow \varepsilon_{1}$, the two extreme values coincide at the inflection point $\omega^{* *}=2.6255$. Besides $\omega_{M}$ and $\omega_{m}$ the equations $\mathcal{F}(\omega ; \varepsilon)=F_{M}$ and $\mathcal{F}(\omega ; \varepsilon)=F_{m}$ both have another solution, denoted by $\tilde{\omega}_{M}$ and $\tilde{\omega}_{m}$, respectively; see Fig. 1 .

Since $F(r)=r \bar{f} / 2$ with constant $\bar{f}, F(r)$ reaches its maximum at the wall $r=1$. This maximum, called $F_{W}(=\bar{f} / 2)$, exceeds the maximum $F_{M}$, if $\bar{f}>2 F_{M}$. Denote the critical pressure gradient by $\bar{f}_{\text {crit }}:=2 F_{M}$ and let $0<\varepsilon<\varepsilon_{1}$. Then in supercritical flow (i.e. $\bar{f}>\bar{f}_{\text {crit }}$ ) equation (3.10) has

- one solution if $0 \leq F<F_{m}$,

- three solutions if $F_{m}<F<F_{M}$,

- one solution if $F_{M}<F \leq F_{W}$;

see Fig. 1. Let $r_{M}:=2 F_{M} / \bar{f}$, then $r_{M}<1$ in supercritical flow. Consequently, we expect that if $0 \leq r<r_{M}$ then $\omega(r)<\omega_{M}$, whereas if $r_{M}<r \leq 1$ then $\omega(r)>\tilde{\omega}_{M}$. Hence, the steady state velocity gradient suffers a jump at $r=r_{M}$, i.e.

$$
\omega_{M}=\lim _{r \uparrow r_{M}} \omega(r)<\lim _{r \downarrow r_{M}} \omega(r)=\tilde{\omega}_{M},
$$

resulting in a kink in the velocity profile at $r=r_{M}$; see Fig. 2 .

\section{$4 \quad$ Stability analysis}

As the steady state equation (3.2) can have more than one solution, we investigate the stability of the different solutions and we establish which particular solution eventually will be attained. In this section especially the stability question will be discussed. To 


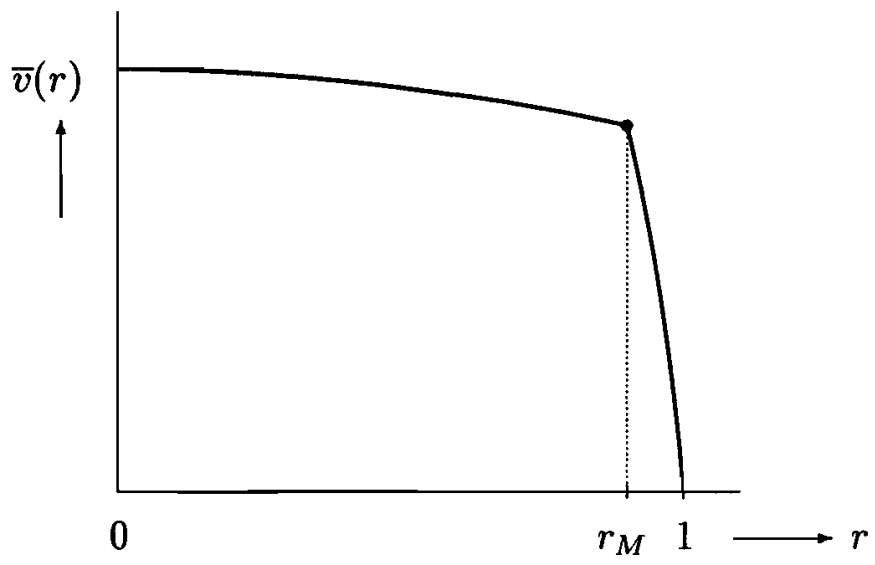

Figure 2 The steady state velocity profile in supercritical flow with a kink at $r=r_{M}$.

investigate the stability properties of any solution $\omega$ we assume that the system is in the steady state at $t=t_{0}$; this will be accomplished by making a special choice for $f(t)$ during the period $0 \leq t \leq t_{0}$ (see (4.4)). The steady state is then slightly perturbed at $t=t_{0}$. The behaviour of the perturbed solution for $t>t_{0}$ determines the stability of the unperturbed solution: If the perturbed solution remains bounded (or tends to zero) then the unperturbed solution is (asymptotically) stable. The definitions of stability of solutions of integrodifferential equations used here are similar to the usual definitions for ordinary differential equations (see [5], [6] and [7]).

To obtain an equation in terms of the total amount of shear, we substitute

$$
-\frac{\partial v}{\partial r}(r, t)=\frac{\partial \Gamma}{\partial t}(r, t)
$$

into (2.26) and find the integrodifferential equation for $\Gamma$

$$
\varepsilon \frac{\partial \Gamma}{\partial t}(r, t)+h(\Gamma(r, t)) e^{-t}+\int_{0}^{t} h(\Gamma(r, t)-\Gamma(r, \tau)) e^{-(t-\tau)} d \tau=\frac{1}{2} r f(t), \quad t>0 .
$$

Since $f(t)$ can be prescribed, we choose $f(t)$ for $t \leq t_{0}$, such that $\partial v / \partial r=-\omega$ (for given $\omega)$ holds exactly for $t \leq t_{0}$. Then the total amount of shear equals

$$
\Gamma(r, t)=\omega(r) t, \quad 0 \leq t \leq t_{0} .
$$

The corresponding function $f=f_{\omega}(t)$ can simply be found by substituting (4.3) into (2.26), yielding

$$
f_{\omega}(t)=\frac{2}{r}\left[\varepsilon \omega(r)+h(\omega(r) t) e^{-t}+\int_{0}^{t} h(\omega(r) \tau) e^{-\tau} d \tau\right], \quad 0 \leq t \leq t_{0} .
$$

Then $\lim _{t \rightarrow \infty} f_{\omega}(t)=\bar{f}$, as occurring in (3.2). As the dependence on $r$ is irrelevant for our stability analysis, we disregard this variable and represent all functions as depending only on $t$. 
To investigate the stability of the steady state associated with a given $\omega$-value, we consider perturbations on this state for $t>t_{0}$. These perturbations can be thought of as due to a short pulse in the pressure $f(t)$ at $t=t_{0}$, causing a small discontinuity in $\Gamma$ (i.e. $\left.\Gamma\left(t_{0}^{+}\right) \neq \Gamma\left(t_{0}^{-}\right)=\omega t_{0}\right)$. From now on we consider only times $t \geq t_{0}$. The perturbation of the velocity gradient is denoted by $\xi$, so that

$$
-\frac{\partial v}{\partial r}\left(t+t_{0}\right)=\omega+\xi(t), \quad t \geq 0 .
$$

Integration with respect to $t$ of $(4.5)$ yields

$$
\Gamma\left(t+t_{0}\right)=\omega t+X(t)+\omega t_{0}, \quad t \geq 0,
$$

where $X$ is defined by

$$
X(t)=X_{0}+\int_{0}^{t} \xi(\tau) d \tau, \quad t \geq 0
$$

Here, $X_{\mathbf{0}}$ stands for the jump in $\Gamma$ at $t=t_{0}$. We replace $t$ by $t+t_{0}$ in (4.2), substitute (4.3) and (4.6) into (4.2), use relation (3.2) for $\omega$ and represent the function $h$ by a Taylor expansion around $\omega t$. Thus, we find an integrodifferential equation for $X$, holding for $t>0$,

$$
\frac{d X}{d t}(t)=B\left(t+t_{0}\right)-A\left(t+t_{0}\right) X(t)+\int_{0}^{t} a(t-\tau) X(\tau) d \tau+\mathcal{G}\left(X(.), t, t_{0}\right)
$$

with

$$
\begin{array}{ll}
a(t)=\frac{1}{\varepsilon} h^{\prime}(\omega t) e^{-t}, & b(t)=-\int_{t}^{\infty} a(\tau) d \tau, \\
A(t)=a(t)+\int_{0}^{t} a(\tau) d \tau, & B(t)=\frac{r}{2 \varepsilon}[f(t)-\bar{f}]-\omega b(t),
\end{array}
$$

and

$$
\begin{aligned}
\mathcal{G}\left(X(.), t, t_{0}\right)=-\frac{1}{2 \varepsilon}\{ & h^{\prime \prime}\left(\theta_{1}\right) e^{-\left(t+t_{0}\right)} X^{2}(t)+X^{2}(t) \int_{t}^{t+t_{0}} h^{\prime \prime}\left(\theta_{2}\right) e^{-\tau} d \tau \\
& \left.+\int_{0}^{t} h^{\prime \prime}\left(\theta_{3}\right)[X(t)-X(\tau)]^{2} e^{-(t-\tau)} d \tau\right\} .
\end{aligned}
$$

Here, the prime denotes differentiation with respect to the argument. The functions $\theta_{i}, i=1,2,3$, are given by $\theta_{1}(t)=\omega\left(t+t_{0}\right)+\kappa_{1} X(t), \theta_{2}(t, \tau)=\omega \tau+\kappa_{2} X(t)$ and $\theta_{3}(t, \tau)=\omega(t-\tau)+\kappa_{3}[X(t)-X(\tau)]$, for some $\kappa_{i}, i=1,2,3$, between 0 and 1 . Since $a \epsilon L^{1}(0, \infty)$ and $A$ is locally integrable, the solution $X$ of $(4.8)$ is known to exist locally (see Driver [8]). The functional $\mathcal{G}$ in $(4.10)$ is of 'higher order', which means that

$$
\mathcal{G}(0, t)=0, \quad \text { and } \quad \mathcal{G}(X, t)=\mathcal{O}(\|X\|), \quad\|X\| \rightarrow 0 .
$$


Hence, $\mathcal{G}$ is locally Lipschitz continuous in $X$, implying that the initial value $X(0)=X_{0}$ determines the solution uniquely. Under these conditions Grossman and Miller [5] proved that the stability of $(4.8)$ is determined by the stability of its linear form (with $\mathcal{G}=0$ )

$$
X^{\prime}(t)=B\left(t+t_{0}\right)-A\left(t+t_{0}\right) X(t)+\int_{0}^{t} a(t-\tau) X(\tau) d \tau, \quad X(0)=X_{0} .
$$

The resolvent $R$ associated with this linear equation, is defined as the unique solution of

$$
R^{\prime}(t)=-A_{\infty} R(t)+\int_{0}^{t} a(t-\tau) R(\tau) d \tau, \quad t>0, \quad R(0)=1
$$

Here, $A_{\infty}$ is defined as the limit for $t \rightarrow \infty$ of the function $A$, i.e. $A_{\infty}=-b(0)$. We shall show that the stability properties of (4.12) depend on the behaviour of $R$ and its derivative. If $R$ is locally integrable, then equation (4.12) is equivalent to its variation of constants form

$$
X(t)=R(t) X_{0}+\int_{0}^{t} R(t-\tau)\left[B\left(\tau+t_{0}\right)+C\left(\tau+t_{0}\right) X(\tau)\right] d \tau, \quad t \geq 0,
$$

as $A$ and $a$ are locally integrable (see [5] for details). Here, the function $C$ is defined by $C(t)=A_{\infty}-A(t)$ and belongs to $L^{\mathbf{1}}(0, \infty)$. Since we are interested in the behaviour of $\xi$, we differentiate equation (4.14) with respect to $t$ and obtain

$$
\xi(t)=\rho(t) X_{0}+B\left(t+t_{0}\right)+C\left(t+t_{0}\right) X(t)+\int_{0}^{t} \rho(t-\tau)\left[B\left(\tau+t_{0}\right)+C\left(\tau+t_{0}\right) X(\tau)\right] d \tau .(4
$$

Here, the integral resolvent $\rho$ is defined as the derivative of $R$ by the relation

$$
R(t)=1+\int_{0}^{t} \rho(\tau) d \tau
$$

and satisfies (see Grossman and Miller [7])

$$
\rho(t)=b(t)+\int_{0}^{t} b(t-\tau) \rho(\tau) d \tau
$$

Since $b(t) \epsilon L^{1}(0, \infty)$, the Paley-Wiener theorem $\left[9\right.$, p. 60] states that $\rho(t) \epsilon L^{1}(0, \infty)$ if and only if

$$
1-b^{*}(z) \neq 0, \quad \operatorname{Re} z \geq 0,
$$

where $b^{*}$ denotes the Laplace transform of the function $b$. Analogous to Miller [6], we show that the integrability of the integral resolvent is crucial.

Proposition 4.1 If $\rho(t) \epsilon L^{1}(0, \infty)$ and $B(t) \epsilon L^{1}(0, \infty)$, then the solution $X(t)$ of (4.14) is bounded, whereas its derivative $\xi(t)$ is bounded and tends to zero as $t \rightarrow \infty$, independent of $t_{0}$. 
Proof: If $\rho(t)$ and $B(t) \epsilon L^{1}(0, \infty)$, then the functions $R(t)$ (see (4.16)) and $\int_{0}^{t}\left|B\left(\tau+t_{0}\right)\right| d \tau$ are bounded. This implies that there exist positive constants $M_{1}$ and $M_{2}$ such that the solution $X$ of (4.14) satisfies

$$
|X(t)| \leq M_{1}\left|X_{0}\right|+M_{2}+M_{1} \int_{0}^{t}\left|C\left(\tau+t_{0}\right)\right||X(\tau)| d \tau, \quad t \geq 0 .
$$

By applying Gronwall's lemma to this equation we obtain

$$
|X(t)| \leq\left(M_{1}\left|X_{0}\right|+M_{2}\right) \exp \left\{M_{1} \int_{0}^{t}\left|C\left(\tau+t_{0}\right)\right| d \tau\right\}, \quad t \geq 0
$$

Since $C(t) \epsilon L^{1}(0, \infty), X(t)$ is bounded for all $t \geq 0$, independent of $t_{0}$. Hence, there exists a positive constant $M_{3}$ such that the derivative $\xi(t)$ satisfying (4.15), can be estimated by

$$
\begin{aligned}
|\xi(t)| \leq|\rho(t)|\left|X_{0}\right|+\left|B\left(t+t_{0}\right)\right|+M_{3}\left|C\left(t+t_{0}\right)\right| & \\
& \quad+\int_{0}^{t}|\rho(t-\tau)|\left(\left|B\left(\tau+t_{0}\right)\right|+M_{3}\left|C\left(\tau+t_{0}\right)\right|\right) d \tau .
\end{aligned}
$$

Since the convolution of two $L^{1}$-functions results in a $L^{1}$-function (by Fubini's theorem), the last term of (4.21) is bounded, implying that $\xi(t)$ is bounded for all $t \geq 0$, independent of $t_{0}$. Moreover, $\rho(t)$ (see (4.17)) and the last term of (4.21) tend to zero as $t \rightarrow \infty$, as the convolution of an $L^{1}$-function with a function that tends to zero yields a function that also tends to zero as $t \rightarrow \infty[9, \mathrm{p} .59]$. Thus, $\xi(t)$ tends to zero as $t \rightarrow \infty$, independent of $t_{0}$.

Lemma 4.1 If $\rho(t) \notin L^{1}(0, \infty)$ then the solutions $X(t)$ and $\xi(t)$ are unbounded.

Proof: This is trivial since $\rho(t) \notin L^{1}(0, \infty)$ implies that $R(t)$ is unbounded. Hence, the solution $X$ of (4.14) is unbounded, which implies by (4.15) that $\xi$ is unbounded.

Using these results in the Paley-Wiener theorem, we present the necessary and sufficient condition for stability of the solution $\omega$, expressed in terms of the function $G_{\omega}$, defined by

$$
G_{\omega}(z)=\varepsilon \omega z+J(\omega)-J\left(\frac{\omega}{1+z}\right), \quad \operatorname{Re} z>-1
$$

\section{Lemma 4.2}

- If $G_{\omega}(z) \neq 0$ for Re $z \geq 0, z \neq 0$, and $G_{\omega}^{\prime}(0) \neq 0$, then the solution $\omega$ is asymptotically stable;

- if $G_{\omega}(z)$ has a zero $z_{0}$ with Re $z_{0}>0$, then the solution $\omega$ is unstable. 
Proof: Since $b^{\prime}(t)=a(t)$ and $b(0)=-A_{\infty}$, the Laplace transform of the function $b$ is equal to $b^{*}(z)=\left(a^{*}(z)-A_{\infty}\right) / z$. Hence, condition (4.18) is equivalent to $z-a^{*}(z)+A_{\infty} \neq 0$ for $\operatorname{Re} z \geq 0, z \neq 0$, and $b^{*}(0) \neq 1$. Calculation of $A_{\infty}=J(\omega) / \varepsilon \omega$ and of the Laplace transform $a^{*}$ of the function $a$,

$$
a^{*}(z)=\int_{0}^{\infty} a(t) e^{-z t} d t=\frac{1}{\varepsilon} \int_{0}^{\infty} h^{\prime}(\omega t) e^{-(z+1) t} d t=\frac{1}{\varepsilon \omega} J\left(\frac{\omega}{1+z}\right), \quad \operatorname{Re} z>-1,
$$

turns the condition into $G_{\omega}(z) \neq 0$ for $\operatorname{Re} z \geq 0, z \neq 0$, and $\lim _{z \rightarrow 0} G_{\omega}(z) / z=G_{\omega}^{\prime}(0) \neq 0$.

We investigate the zeros of $G_{\omega}(z)$ in the half-plane $\operatorname{Re} z \geq 0$. The derivative of $G_{\omega}$ equals

$$
G_{\omega}^{\prime}(z)=\omega\left[\varepsilon+\frac{1}{(1+z)^{2}} J^{\prime}\left(\frac{\omega}{1+z}\right)\right], \quad \operatorname{Re} z>-1,
$$

implying that $G_{\omega}^{\prime}(0)=\omega\left(\varepsilon+J^{\prime}(\omega)\right)$. To investigate if there are any zeros of $G_{\omega}$ on the positive real axis we substitute $z=x$, where $x$ is real and positive.

Lemma 4.3 If $0 \leq \omega<\omega_{M}$ or $\omega>\omega_{m}$, the function $G_{\omega}(x)$ increases strictly on the interval $(0, \infty)$.

Proof: Suppose $0 \leq \omega<\omega_{M}$. Then $\omega /(1+x)<\omega<\omega_{M}, x>0$, implies $J^{\prime}(\omega /(1+x))>$ $J^{\prime}\left(\omega_{M}\right)=-\varepsilon$ (see Appendix). Substitution of this inequality into (4.24) yields

$$
G_{\omega}^{\prime}(x)>\varepsilon \omega\left[1-\frac{1}{(1+x)^{2}}\right]>0, \quad x>0, \quad 0 \leq \omega<\omega_{M} .
$$

Suppose $\omega>\omega_{m}$. For $x \epsilon\left[0, \omega / \omega_{m}-1\right)$ or $x \epsilon\left(\omega / \omega_{M}-1, \infty\right)$, the variable $\omega /(1+x)$ exceeds $\omega_{m}$ or remains below $\omega_{M}$, respectively, implying that $J^{\prime}(\omega /(1+x))>-\varepsilon$ in both cases. Hence, inequality (4.25) also holds for $x \epsilon\left[0, \omega / \omega_{m}-1\right) \cup\left(\omega / \omega_{M}-1, \infty\right), \omega>\omega_{m}$. For $\omega / \omega_{m}-1 \leq x \leq \omega / \omega_{M}-1$, we can estimate $G_{\omega}^{\prime}(x)$ by

$$
\begin{aligned}
& \omega G_{\omega}^{\prime}(x)=\varepsilon \omega^{2}+\left(\frac{\omega}{1+x}\right)^{2} J^{\prime}\left(\frac{\omega}{1+x}\right)>\varepsilon\left(\omega^{2}-\omega_{m}^{2}\right)>0 \\
& \omega_{M} \leq \frac{\omega}{1+x} \leq \omega_{m}, \quad \omega>\omega_{m},
\end{aligned}
$$

since the function $\omega^{2} J^{\prime}(\omega)$ decreases strictly if $\omega>\omega^{*}$ (see Appendix).

Lemma 4.4 For $x>0$ the function $G_{\omega}(x)$ has

- no positive zeros if $0 \leq \omega<\omega_{M}$ or $\omega>\omega_{m}$,

- at least one positive zero if $\omega_{M}<\omega<\omega_{m}$. 
Proof: Suppose $0 \leq \omega<\omega_{M}$ or $\omega>\omega_{m}$. Then Lemma 4.3 and $G_{\omega}(0)=0$ imply that $G_{\omega}(x)$ is positive for $x>0$. For $\omega_{M}<\omega<\omega_{m}, G_{\omega}^{\prime}(0)$ is negative. Since $G_{\omega}(x)=\varepsilon \omega x[1+\mathcal{O}(1)], x \rightarrow \infty$, and $G_{\omega}(0)=0$, the function $G_{\omega}$ must have at least one positive zero.

Lemma 4.5 The function $G_{\omega}(z)$ has no complex zeros in the quadrant Re $z>0, \operatorname{Im} z>0$.

Proof: Write $z=x+i y=:(\xi-1)+i \xi \nu$, with $\xi>1$ and $\nu>0$, and define $\sigma:=\xi / \omega, \sigma>0$. The function $G_{\omega}(z)$ has a real part

$$
\operatorname{Re} G_{\omega}(z)=\varepsilon \frac{\xi}{\sigma}(\xi-1)+J\left(\frac{\xi}{\sigma}\right)-\sigma\left[\left(1+\nu^{2}\right) T_{1}(\nu, \sigma)+T_{3}(\nu, \sigma)\right]
$$

and an imaginary part

$$
\operatorname{Im} G_{\omega}(z)=\frac{\nu \xi^{2}}{\sigma}\left\{\varepsilon+\left(\frac{\sigma}{\xi}\right)^{2}\left[\left(1+\nu^{2}\right) T_{1}(\nu, \sigma)-T_{3}(\nu, \sigma)\right]\right\},
$$

where

$$
T_{n}(\nu, \sigma)=\int_{0}^{\infty} \frac{t^{n} e^{-\sigma t}}{\left(1-\nu^{2}+t^{2}\right)^{2}+4 \nu^{2}} d t, \quad n=1,3 .
$$

By elimination of $\varepsilon$ from the two equations $\operatorname{Re} G_{\omega}(z)=0$ and $\operatorname{Im} G_{\omega}(z)=0$, we find

$$
R(\xi, \nu, \sigma):=(2 \xi-1)\left(1+\nu^{2}\right) T_{1}(\nu, \sigma)+T_{3}(\nu, \sigma)-\frac{\xi}{\sigma} J\left(\frac{\xi}{\sigma}\right)=0 .
$$

Thus, $G_{\omega}(z)$ has a complex zero if and only if the two equations $R(\xi, \nu, \sigma)=0$ and $\operatorname{Im} G_{\omega}(z)=0$ have a solution. Numerical calculations reveal that the equation

$$
\left(1+\nu^{2}\right) T_{1}(\nu, \sigma)-T_{3}(\nu, \sigma)=0
$$

has a unique solution $\nu=\nu_{0}(\sigma)$, plotted in Fig. 3, while

$$
\left(1+\nu^{2}\right) T_{1}(\nu, \sigma)-T_{3}(\nu, \sigma)>0, \quad \text { if } \quad \nu>\nu_{0}(\sigma)
$$

Also by numerical calculation we find that, for fixed $\xi>1$, the equation $R(\xi, \nu, \sigma)=0$ has a unique solution $\nu=\nu_{\xi}(\sigma)$, as plotted in Fig. 3 for $\xi=1,2,3,4$. From these calculations it is evident that $\nu_{\xi}(\sigma)>\nu_{0}(\sigma)$ for $\xi>1$ and $\sigma>0$. Hence, $R$ vanishes only in the region $\nu>\nu_{0}$, whereas $\operatorname{Im} G_{\omega}(z)>0$ in this region, according to (4.32). This implies that there are no triples $(\xi, \nu, \sigma)$ such that the corresponding values of $z$ and $\omega$ satisfy $\operatorname{Re} G_{\omega}(z)=0$ and $\operatorname{Im} G_{\omega}(z)=0$.

Conclusion We conclude this section by recapitulating the results concerning the stability of the unperturbed solution $\omega$. We proved that

1. steady states with $0 \leq \omega<\omega_{M}$ or $\omega>\omega_{m}$ are asymptotically stable,

2. steady states with $\omega_{M} \leq \omega \leq \omega_{m}$ are unstable.

Moreover, the stability analysis implies that $\lim _{t \rightarrow \infty}-\partial v(r, t) / \partial r=\omega(r)$ exists (as we needed in the proof of Proposition 3.1). 


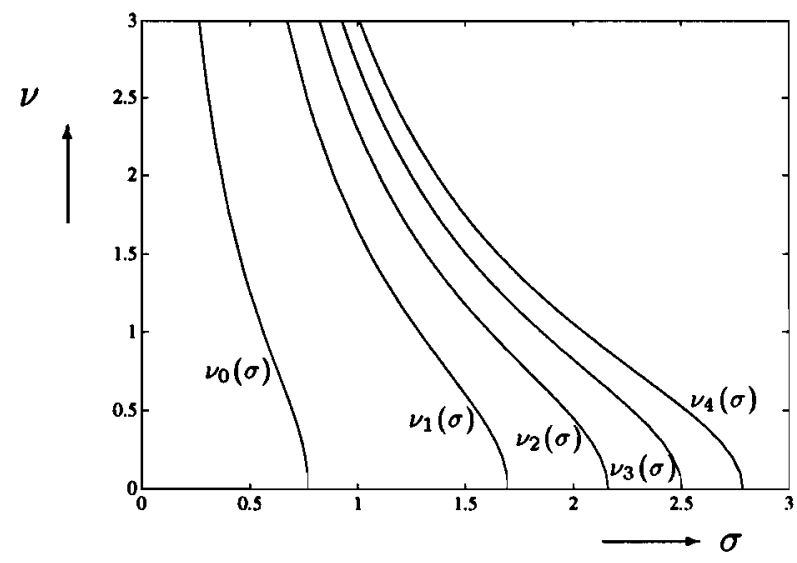

Figure 3 The curves $\nu=\nu_{0}(\sigma)$ and $\nu=\nu_{\xi}(\sigma)$, for $\xi=1,2, \ldots$ in the $(\sigma, \nu)$-plane.

\section{Some numerical results}

In this section we present some results of numerical calculations based on the integrodifferential equation (4.2). These results will confirm the conclusions of our stability analysis and will provide extra information on such phenomena as spurt, shape memory and hysteresis. When starting up the flow from rest, given a fixed value $\bar{f}$ for the pressure gradient $f(t)$, the velocity gradient will attain a steady value $\omega(r)$, where $\omega$ lies either below $\omega_{M}$ or above $\omega_{m}$, whereas steady states with $\omega$ between $\omega_{M}$ and $\omega_{m}$ are unstable and will never be attained.

Consider experiments in which the flow is initially in a steady state, reached at time $t=t_{0}$, corresponding to a forcing $\bar{f}_{0}$, and the forcing is suddenly changed to $\bar{f}=\bar{f}_{0}+\Delta \bar{f}$. If $\Delta \bar{f}$ is positive, we call this process loading, otherwise unloading. Suppose $|\Delta \bar{f}|$ is sufficiently small to reach another steady state after some time. The outcome of this experiment depends on the initial state $\bar{f}_{0}$. Malkus et al. [2] discussed the quasi-static loading-unloading cycle, where the load is gradually increased from $\bar{f}=0$ up to $\bar{f}_{\max }>$ $\bar{f}_{\text {crit }}$, followed by an unloading sequence until the initial state $\bar{f}=0$ is reached. During the first part of the loading, where $\bar{f}<\bar{f}_{\text {crit }}=2 F_{M}$ (subcritical flow) the entire flow is classical: The velocity gradient satisfies $\omega(r)<\omega_{M}$ and is continuous in $r$ for all $r \epsilon[0,1]$. In Fig. 4 the stationary volumetric flow $\bar{Q}$ (calculated by means of $(2.33)$ with $-\partial v / \partial r=\omega$ ) is depicted as a function of $\bar{f}$; the curve $\mathrm{AB}$ corresponds to the subcritical flow. When the flow becomes supercritical $\left(\bar{f} \geq \bar{f}_{\text {crit }}\right)$ a kink in the velocity profile forms at the wall, moving away from the wall to a position $r_{M}=2 F_{M} / \bar{f}$, for further increasing $\bar{f}$. The high values of the velocity gradient near the wall cause an enormous increase of the volumetric rate (from $\mathrm{B}$ to $\mathrm{C}$ in Fig. 4). For $\bar{f}=\bar{f}_{\max }$ the spurt layer $r^{*} \leq r \leq 1$, where $r^{*}=2 F_{M} / \bar{f}_{\max }$, is of maximum thickness. From this point we start to unload. At first the spurt layer remains fixed between $r=r^{*}=2 F_{M} / \bar{f}_{\max }$ and $r=1$; this phenomenon called shape memory corresponds to the path from $\mathrm{C}$ to $\mathrm{D}$ in Fig. 4. During this unloading the magnitude of the shear stress $\left|T_{r z}\right|=F$ at $r^{*}$ decreases according to $F^{*}:=F\left(r^{*}\right)=r^{*} \bar{f} / 2=F_{M} \bar{f} / \bar{f}_{\max }$. If $F^{*}$ falls below $F_{m}$ (i.e. if $\bar{f}<F_{m} \bar{f}_{\max } / F_{M}$ ), the layer position $r=r_{m}:=2 F_{m} / \bar{f}$ moves back towards the wall for further decreasing $\bar{f}$. This loss of shape memory corresponds to 
the path from $\mathrm{D}$ to $\mathrm{E}$ in Fig. 4. The spurt layer disappears for $\bar{f}=2 F_{m}$ (i.e. $r_{m}=1$, which corresponds to point $\mathrm{E}$ in Fig. 4$)$ and after that $\left(\bar{f}<2 F_{m}\right)$ the flow becomes entirely classical again. In the final unloading path $\mathrm{EA}$, the flow is classical and this path coincides with the initial part of the loading curve. The phenomenon that no part of the loading curve in Fig. 4 is retraced until the flow has become entirely classical again, is typical for hysteresis.

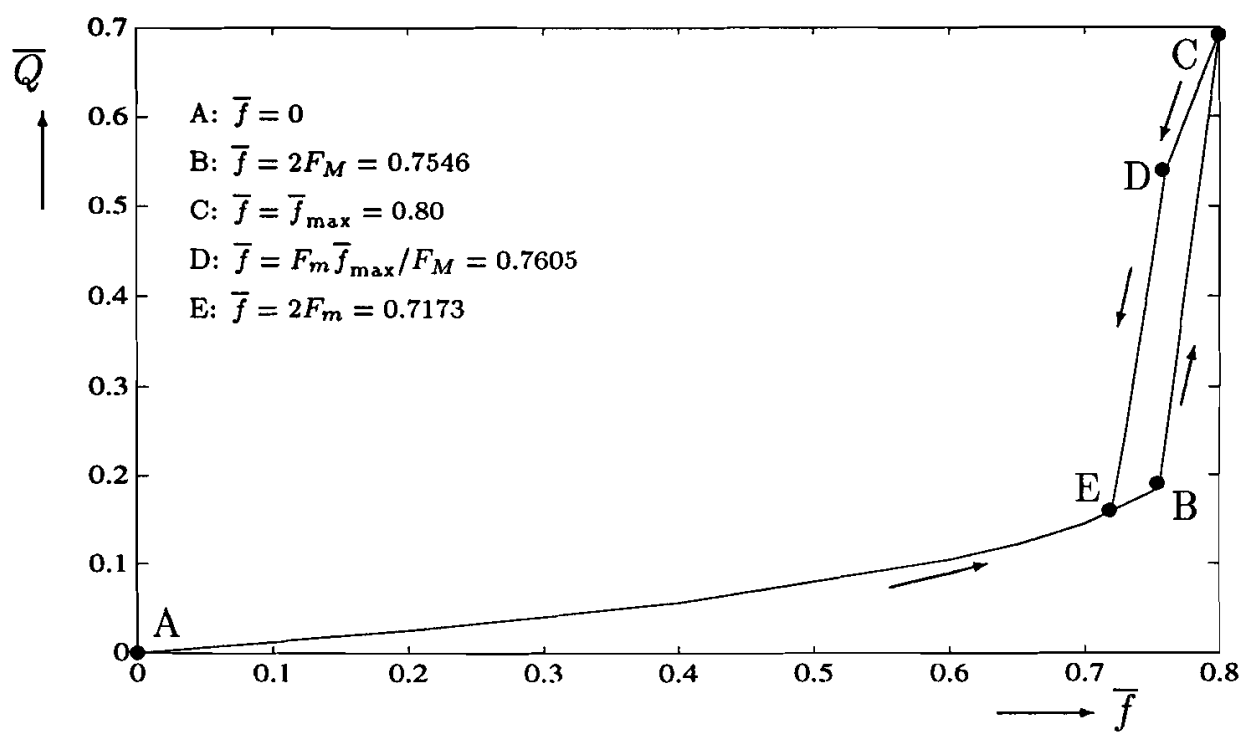

Figure 4 Hysteresis under cyclic load. The stationary volumetric flow $\bar{Q}$ versus the loading $\bar{f}$, with $\varepsilon=0.02$ and $\bar{f}_{\max }=0.80$.

The loading-unloading behaviour of the flow as described above, is confirmed by the results of numerical calculations of the velocity gradient. At $t=0$ the velocity profile is parabolic

$$
v(r, 0)=\frac{f(0)}{4 \varepsilon}\left(1-r^{2}\right), \quad 0 \leq r \leq 1,
$$

as can be obtained by letting $t \downarrow 0$ in (4.2). For $t>0$ the velocity gradient $-\partial v / \partial r=\partial \Gamma / \partial t$ is obtained by computing the total amount of shear $\Gamma(r, t)$ as solution of equation (4.2) for a fixed value of $r$ and the pressure gradient $f(t)$ prescribed. We solve this differential equation with initial condition $\Gamma(r, 0)=0$ by using Euler's forward discretisation method with fixed step length. The integral is approximated by the trapezoidal rule. Since $\varepsilon$ is small, the term $\varepsilon \partial \Gamma / \partial t$ dominates the initial response of the fluid, implying that the step length must be sufficiently small. Because of the hereditary effect we have to use all values of $\Gamma(r, \tau), \tau \leq t$, to compute $\Gamma(r, t)$. However, for $t$ sufficiently large $(t>T)$ the integral can be approximated by the integral over the interval $[t-T, t]$, since

$$
\int_{0}^{t} h(\gamma) e^{-(t-\tau)} d \tau=\int_{t-T}^{t} h(\gamma) e^{-(t-\tau)} d \tau+O\left(e^{-T}\right), \quad T \rightarrow \infty
$$


By choosing $T=20$, we prevent the exponential growth of the number of calculation steps for increasing time $t$.

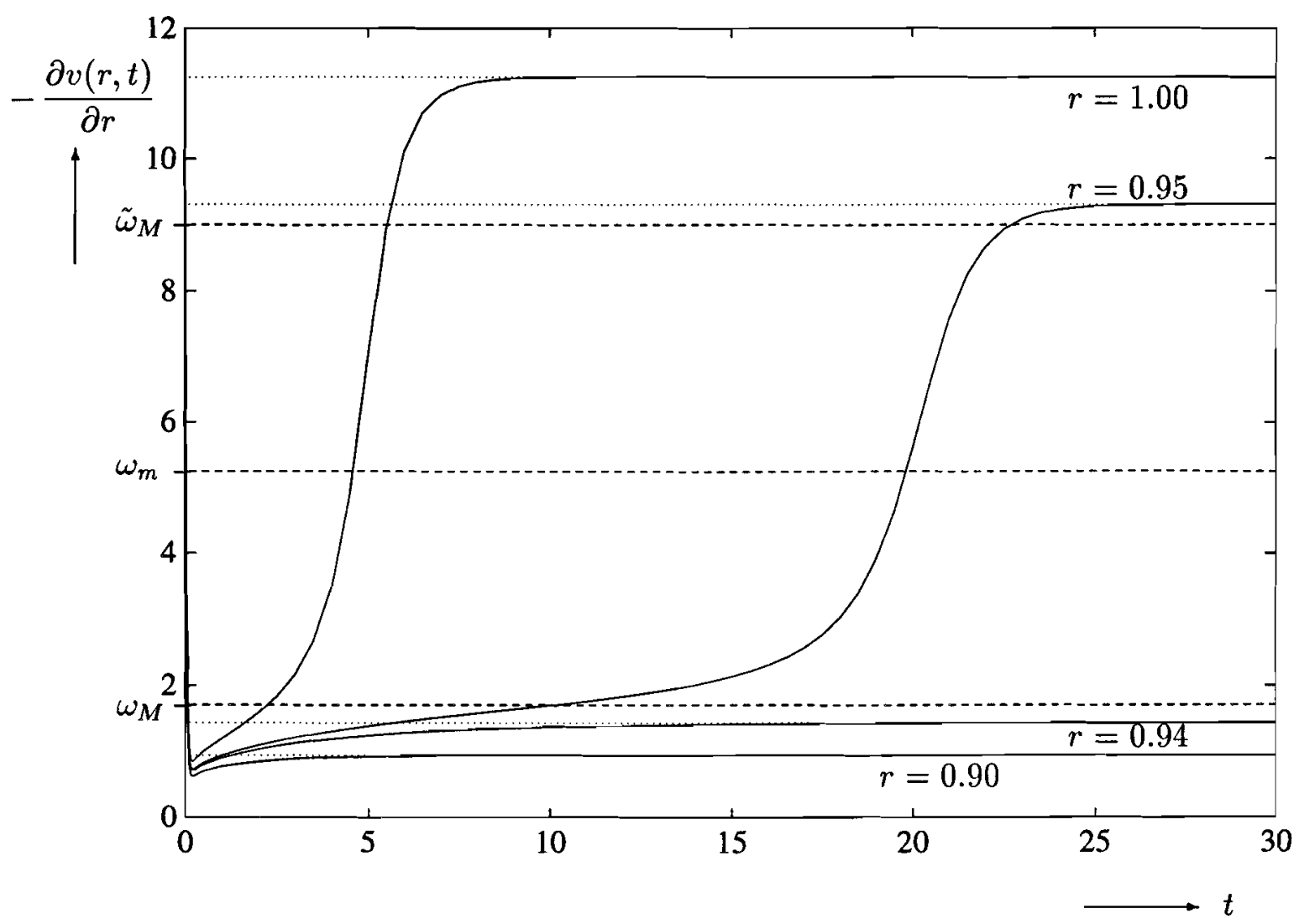

Figure 5 The velocity gradient $-\partial v(r, t) / \partial r$ for $\varepsilon=0.02$ and $f(t)=0.8 H(t)$, computed by Euler's forward discretisation method with step length $h_{t}=0.005$. The steady state velocity gradient jumps at $r=r_{M}=0.9432$.

The total amount of shear is computed for a prescribed pressure gradient of the form

$$
f(t)=\bar{f} H(t),
$$

and we take $\varepsilon=0.02\left(<\varepsilon_{1}\right)$ and $\bar{f}=0.8>\bar{f}_{\text {crit }}=2 F_{M}=0.7546$ to let the flow be supercritical. In Fig. 5 the computed velocity gradient as a function of $t$ is plotted for some values of $r$. We observe that the velocity gradient approaches a steady state value, equal to solution $\omega$ numerically computed from $\varepsilon \omega+J(\omega)=r \bar{f} / 2$ and represented by the dotted lines in Fig. 5. As long as $0 \leq r<r_{M}=2 F_{M} / \bar{f}(=0.9432$ for $\varepsilon=0.02$ and $\bar{f}=0.8)$, the steady state value $\omega(r)$ lies between 0 and $\omega_{M}$. When $r>r_{M}$, the steady state velocity gradient jumps from a value below $\omega_{M}$ to a value that exceeds $\tilde{\omega}_{M}\left(>\omega_{m}\right)$; see Fig. 1. Hence, for $r<r_{M}$ the steady state solution $\omega$ satisfies $\omega(r)<\omega_{M}=1.7063$, whereas for $r>r_{M}$ it satisfies $\omega(r)>\tilde{\omega}_{M}=9.0094$; see also Fig. 8a. 


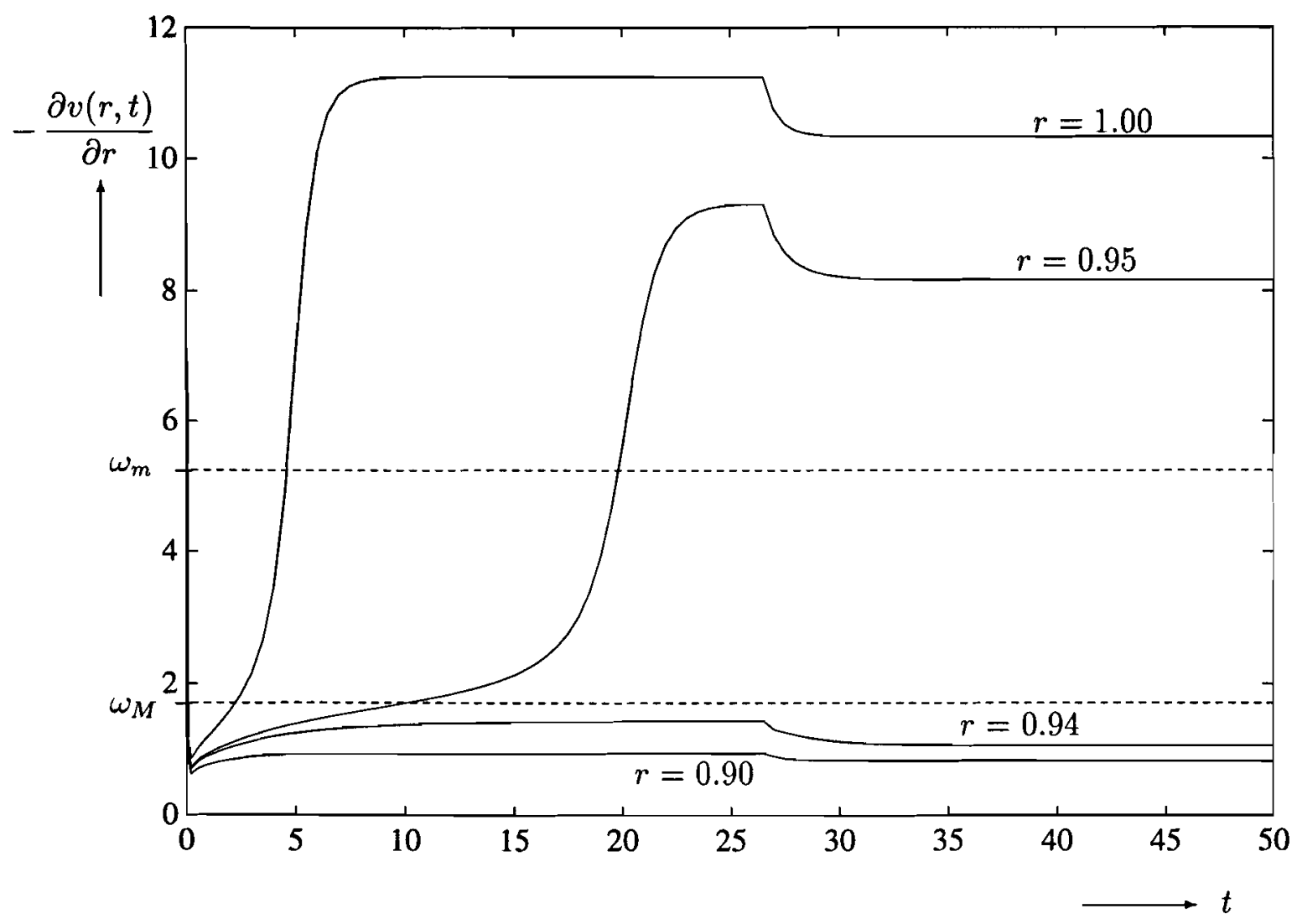

Figure 6 The velocity gradient $-\partial v(r, t) / \partial r$ for $\varepsilon=0.02$ under a changing load $f(t)=0.80 H(t)-0.02 H\left(t-t_{0}\right)$ at $t_{0}=27$, computed by Euler's forward discretisation method with step length $h_{t}=0.005$. Both steady state velocity gradients jump at $r=r_{M}=0.9432$.

The process of unloading is numerically implemented by prescribing the pressure gradient as

$$
f(t)=\bar{f}_{0} H(t)+\left(\bar{f}_{1}-\bar{f}_{0}\right) H\left(t-t_{0}\right),
$$

where $t_{0}$ is sufficiently large to achieve a steady state at $t=t_{0}$. We take $\varepsilon=0.02, \bar{f}_{0}=0.80$ and $t_{0}=27$. To account for the phenomenon of shape memory, related to a position between the points $\mathrm{C}$ and $\mathrm{D}$ in Fig. 4 , we choose $\bar{f}_{1}=0.78>F_{m} \bar{f}_{0} / F_{M}=0.7605$. The numerically computed solution following from this change of load is plotted in Fig. 6 . We observe that after the load has changed from $\bar{f}_{0}=0.80$ to $\bar{f}_{1}=0.78$, the jump in the steady state velocity gradient is still situated between $r=0.94$ and $r=0.95$, implying that the position of the spurt layer remains fixed at the maximum position $r^{*}=2 F_{M} / \bar{f}_{0}=0.9432$. This is called shape memory, because the spurt layer is unchanged, regardless of the change in loading. The set of $\omega(r)$ values (for $r \epsilon[0,1]$ ) can be read off from Fig. 8b. 


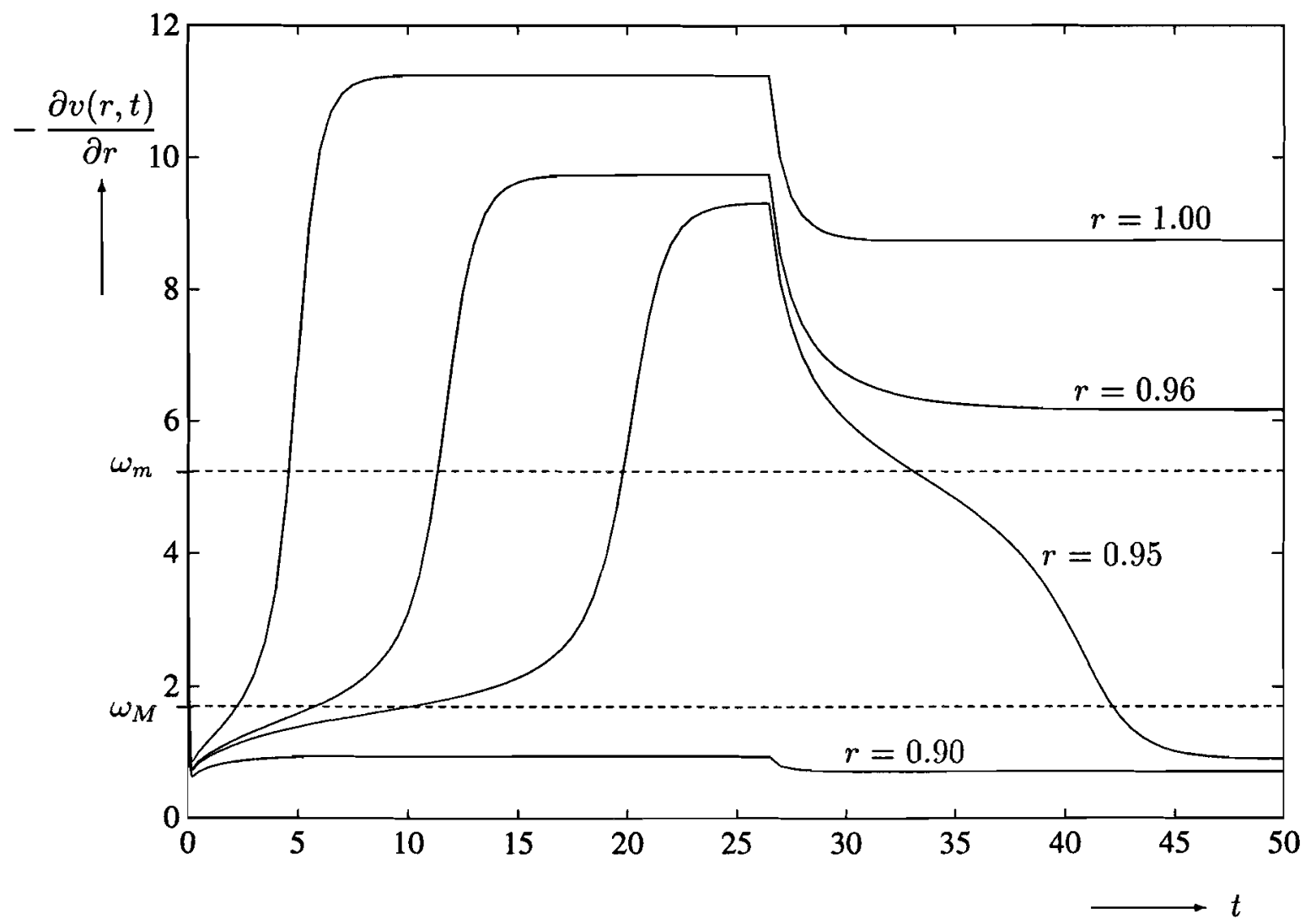

Figure 7 The velocity gradient $-\partial v(r, t) / \partial r$ for $\varepsilon=0.02$ under a changing load $f(t)=0.80 H(t)-0.05 H\left(t-t_{0}\right)$ at $t_{0}=27$, computed by Euler's forward discretisation method with step length $h_{t}=0.005$. The second steady state velocity gradient jumps at $r=r_{m}=0.9564$.

The choice $\bar{f}_{1}=0.75>2 F_{m}=0.7173$ corresponds to a position between the points $\mathrm{D}$ and $\mathrm{E}$ in Fig. 4, where the kink forms at $r^{*}=2 F_{m} / \bar{f}_{1}=0.9564$. In Fig. 7 the solution for this particular loading is presented. We observe that when the load is equal to $\bar{f}_{1}=0.75$, the jump in the steady state velocity gradient occurs between $r=0.95$ and $r=0.96$, which is closer to the wall than its position for $\bar{f}_{0}=0.80$. This moving back of the spurt layer position to the wall corresponds to loss of shape memory. As shown in Fig. 8c, the jump in the velocity gradient is now from $\omega_{m}$ to the value $\tilde{\omega}_{m}<\omega_{M}$.

We conclude that the numerical calculations support our expectations concerning the occurrence and the specific behaviour of a jump in the velocity gradient under a loadingunloading cycle, as expressed in the beginning of this section. 


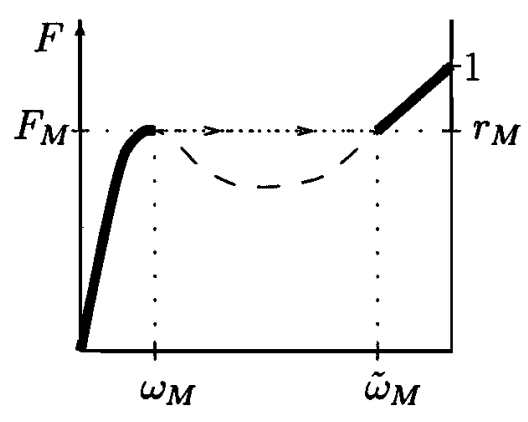

a Loading with $\bar{f}>\bar{f}_{\text {crit }}=2 F_{M}$.

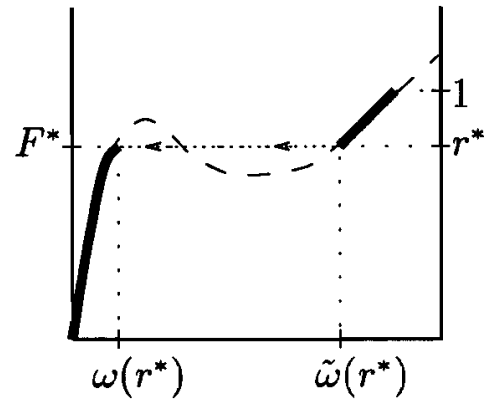

b Unloading with $\bar{f}_{0} \geq \bar{f}_{\text {crit }}$ and $F_{m} \bar{f}_{0} / F_{M}<\bar{f}_{1}<\bar{f}_{0}$.

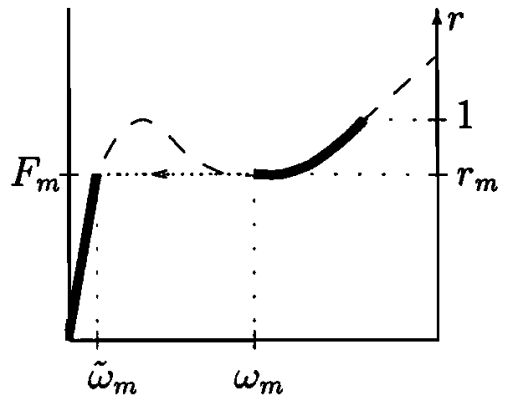

c Unloading with $\bar{f}_{0}>\bar{f}_{\text {crit }}$ and $2 F_{m}<\bar{f}_{1}<F_{m} \bar{f}_{0} / F_{M}$.

Figure 8 The set of $\omega$-values for different values of the stationary pressure gradient $\bar{f}$.

\section{Conclusions}

Stability analysis and numerical simulations have been used to analyse the Poiseuille flow of a KBKZ-fluid supplied with an extra viscous term. This fluid model describes the behaviour of highly elastic polymeric fluids. The addition of a viscous term, leading to a nonmonotonic constitutive behaviour, is essential in this analysis: A kink in the velocity profile, due to a jump in the steady state velocity gradient, provides an explanation of the spurt phenomenon. Hence, internal material properties of the fluid itself account for the spurt phenomenon and not a global external effect as 'wall slip'. Since in industrial practice this spurt effect distorts the extrudate by forming a pattern of irregularities at its surface, a good estimate of the critical value of the pressure gradient (or the associated critical stationary volumetric flow rate) beyond which spurt occurs, is of great practical value.

The aspect which distinguishes our approach from that of [2], where a differential equation is used, is that we analysed a nonlinear viscoelastic constitutive equation containing a memory integral, leading to a nonlinear integrodifferential equation. Recapitulating our main results we proved by analytical means that

- for $t \rightarrow \infty$, the flow reaches a steady state;

- if the stationary pressure gradient exceeds a critical value, equation (3.2) has three distinct solutions for the steady state velocity gradient $\omega(r)$ for a certain range of radial coordinates $r$;

- a steady state $\omega$ with $\omega_{M} \leq \omega \leq \omega_{m}$ is unstable;

- steady states $\omega$ with $0 \leq \omega<\omega_{M}$ and $\omega>\omega_{m}$ are stable.

Numerical results showed how and under which conditions the different stable states are attained. This strongly depends on the radial position and the deformation history of the fluid. In this respect, our calculations reveal that for supercritical flow in the loading 
phase, the flow reaches its steady state gradually as long as $r<r_{M}$ (i.e. $F(r)<F_{M}$ ) (see e.g. Fig. 5). However, for $r>r_{M}$ one observes a rather small time interval in which the velocity gradient jumps from a value below $\omega_{M}$ to a value that exceeds $\omega_{m}$, after which the flow becomes gradually stationary. Since the latter $\omega$-value is much larger than the first one, a kink in the velocity profile appears at $r=r_{M}$. In the layer near the wall, the so-called spurt layer, the magnitude of the velocity gradient is very large, which causes an enormous increase of the volumetric flow rate. Since in our analysis the no-slip boundary condition $\bar{v}(1)=0$ is maintained (see Fig. 2), wall slip can not account for this effect.

In the unloading phase the following peculiarities are observed:

- As long as the unloading is small enough, the spurt layer remains fixed; this effect is referred to as shape memory;

- the decrease of the velocity gradient is at first rather fast, after which it becomes gradually stationary again;

- when the unloading step exceeds a certain value, the thickness of the spurt layer starts to decrease and the layer disappears as soon as the flow becomes subcritical again;

- the unloading and loading paths do not coincide, implying the occurrence of hysteresis.

Up to now, the observations listed above are only supported by numerical calculations. A mathematical proof for the qualitative behaviour of the fluid remains for further research. Due to the integrodifferential character of our equations, an analogy with the methods used by Malkus et al. [2] is not possible. However, we expect that a continued study of e.g.

- the relationship between the shear stress and the (first) normal stress difference,

- the asymptotics for small $\varepsilon$-values,

will provide a further insight in the problem under consideration. Hence, one of the aims of our future investigations is to derive an analytical explanation for effects such as shape memory and hysteresis.

Another peculiar effect, not yet mentioned, that is observed in experiments as well as in industrial circumstances, is the occurrence of the so-called 'shark-skin' instabilities at the surface of an extrudate (cf. [10]). These shark-skins occur in extrusion processes before spurt turns up. It is very well possible that these effects, which show a typical time scale behaviour, are associated with the sudden increase of the velocity gradient in the loading phase as depicted in Figs. 5, 6 and 7. This small time scale behaviour is related to the small value of $\varepsilon$. This supports once more the expectation that a study of the asymptotics with respect to $\varepsilon$ will lead to a better understanding of the flow problem under consideration.

\section{Acknowledgement}

We thank Professor J. Boersma for a careful reading of the manuscript and for helpful discussions and comments. 


\section{References}

[1] D.S. Malkus, J.A. Nohel and B.J. Plohr, Dynamics of shear flow of a nonNewtonian fluid, J. Comput. Phys. 87 (1990), 464-487.

[2] D.S. Malkus, J.A. Nohel and B.J. Plohr, Analysis of new phenomena in shear flow of non-Newtonian fluids, SIAM J. Appl. Math. 51 (1991), 899-929.

[3] G.V. Vinogradov, A.Y. Malkin, Y.G. Yanovskit, E.K. Borisenkova, B.V. YARLYKOV AND G.V. BEREZHNAYA, Viscoelastic properties and flow of narrow distribution polybutadienes an polyisoprenes, J. Polymer Sci. Part A-2 10 (1972), 1061-1084.

[4] R.I. Tanner, Engineering rheology, Clarendon Press, Oxford (1988).

[5] S.I. Grossman and R.K. Miller, Perturbation theory for Volterra integrodifferential systems, J. Differential Equations 8 (1970), 457-474.

[6] R.K. Miller, Asymptotic stability properties of linear Volterra integrodifferential equations, J. Differential Equations 10 (1971), 485-506.

[7] S.I. Grossman and R.K. Miller, Nonlinear Volterra integrodifferential systems with $L^{1}$-kernels, J. Differential Equations 13 (1973), 551-566.

[8] R.D. DrIVER, Existence and stability of solutions of a delay-differential system, Arch. Rational Mech. Anal. 10 (1962), 401-426.

[9] R.E.A.C. Paley And N. Wiener, Fourier transforms in the complex domain, Amer. Math. Soc., New York (1954).

[10] J. MolenaAr and R. Koopmans, Modeling polymer melt-flow instabilities, J. Rheol. 38 (1994), 99-109.

[11] M. Abramowitz and I.A. Stegun (eds.), Handbook of mathematical functions, with formulas, graphs and mathematical tables, Dover Publications, New York (1965).

\section{Appendix Properties of $J(\omega)$}

According to Abramowitz and Stegun [11, p. 232], we have the following expansions for the function $J$ defined in (3.9):

$$
J(\omega)=\frac{\log \omega}{\omega}-\frac{C}{\omega}+\frac{\pi}{2} \frac{1}{\omega^{2}}-\frac{\log \omega}{2 \omega^{3}}+O\left(\frac{1}{\omega^{3}}\right), \quad \omega \rightarrow \infty
$$

and

$$
J(\omega)=\omega-6 \omega^{3}+O\left(\omega^{5}\right), \quad \omega \rightarrow 0 .
$$


Here, $C=0.57721 \ldots$ is Euler's constant. The derivative $J^{\prime}$ of the function $J$ is obtained by differentiation inside the integral sign and integration by parts, yielding

$$
J^{\prime}(\omega)=\int_{0}^{\infty} \frac{\tau^{2}-\tau}{1+\omega^{2} \tau^{2}} e^{-\tau} d \tau=\frac{1-L(\omega)}{\omega^{2}}-\frac{J(\omega)}{\omega}
$$

where the integral $L$ is defined by

$$
L(\omega)=\int_{0}^{\infty} \frac{e^{-\tau}}{1+\omega^{2} \tau^{2}} d \tau
$$

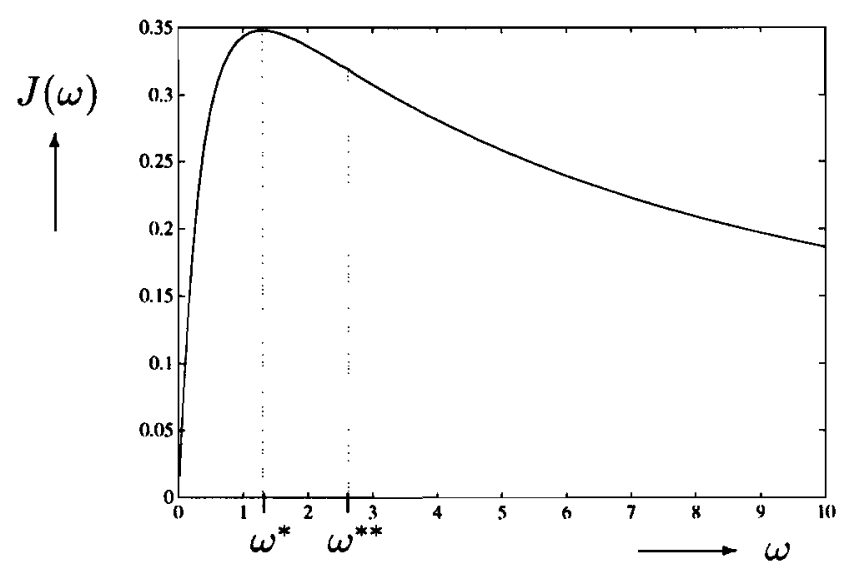

Figure 9 The function $J(\omega)$ for $\omega \geq 0$.

Numerical calculations reveal that on the interval $[0, \infty)$ the function $J$ is non-negative with one maximum $J\left(\omega^{*}\right)=0.34794$ at $\omega^{*}=1.2979$. Its derivative $J^{\prime}$ has one minimum $J^{\prime}\left(\omega^{* *}\right)=-0.02886$ at $\omega^{* *}=2.6255$. Hence, $J^{\prime}$ decreases strictly on the interval $\left[0, \omega^{* *}\right]$ with $J^{\prime}(0)=1$, whereas on $\left[\omega^{* *}, \infty\right)$ it increases strictly to zero. In Fig. 9 the function $J(\omega)$ is plotted. If $0<\varepsilon<\varepsilon_{1}:=-J^{\prime}\left(\omega^{* *}\right)=0.02886$, the function $\varepsilon+J^{\prime}(\omega)$ has two zeros denoted by $\omega_{M}$ and $\omega_{m}$. Let $\omega_{M}<\omega_{m}$, then $\omega^{*}<\omega_{M}<\omega^{* *}<\omega_{m}$. Thus, if $0<\varepsilon<\varepsilon_{1}$,

\begin{tabular}{c|c|c|r|c|c|c}
$\varepsilon$ & $\omega_{M}$ & $\begin{array}{c}F_{M} \\
\left(=\varepsilon \omega_{M}+J\left(\omega_{M}\right)\right)\end{array}$ & $\tilde{\omega}_{M}$ & $\omega_{m}$ & $\begin{array}{c}F_{m} \\
\left(=\varepsilon \omega_{m}+J\left(\omega_{m}\right)\right)\end{array}$ & $\tilde{\omega}_{m}$ \\
\hline 0.010 & 1.4519 & 0.36164 & 25.4974 & 9.9609 & 0.28661 & 0.4703 \\
0.020 & 1.7063 & 0.37730 & 9.0094 & 5.2439 & 0.35867 & 0.9242 \\
0.025 & 1.9463 & 0.38637 & 5.5151 & 3.9248 & 0.38149 & 1.3454
\end{tabular}

Table 1 The zeros $\omega_{M}$ and $\omega_{m}$ of the function $\partial \mathcal{F}(\omega ; \varepsilon) / \partial \omega$ for different values of $\varepsilon\left(0<\varepsilon<\varepsilon_{1}\right)$, the maximum $F_{M}=\mathcal{F}\left(\omega_{M} ; \varepsilon\right)$ and minimum $F_{m}=\mathcal{F}\left(\omega_{m} ; \varepsilon\right)$, and the zeros $\tilde{\omega}_{M}$ of $\mathcal{F}(\omega ; \varepsilon)-F_{M}$ and $\tilde{\omega}_{m}$ of $\mathcal{F}(\omega ; \varepsilon)-F_{m}$, where $\mathcal{F}(\omega ; \varepsilon)=\varepsilon \omega+J(\omega)$.

the function $\mathcal{F}(\omega ; \varepsilon)=\varepsilon \omega+J(\omega)$ has two extreme values, a maximum $F_{M}=\varepsilon \omega+J\left(\omega_{M}\right)$ at $\omega=\omega_{M}$ and a minimum $F_{m}=\varepsilon \omega_{m}+J\left(\omega_{m}\right)$ at $\omega=\omega_{m}$. Moreover, the equations 
$\mathcal{F}(\omega ; \varepsilon)=F_{M}$ and $\mathcal{F}(\omega ; \varepsilon)=F_{m}$ have both (besides $\omega_{M}$ and $\omega_{m}$ ) a second solution, denoted by $\tilde{\omega}_{M}$ and $\tilde{\omega}_{m}$, respectively. Numerical values of $\omega_{M}, \omega_{m}, F_{M}, F_{m}, \tilde{\omega}_{M}$ and $\tilde{\omega}_{m}$ for various $\varepsilon$ are given in Table 1 .

Another useful property is that the function $\omega^{2} J^{\prime}(\omega)$ decreases strictly for $\omega>\omega^{*}$. If $\omega^{*}<\omega<\omega^{* *}$, this is trivial since $d\left\{\omega^{2} J^{\prime}(\omega)\right\} / d \omega=2 \omega J^{\prime}(\omega)+\omega^{2} J^{\prime \prime}(\omega)<0$. By representing $J^{\prime \prime}$ in terms of $J$ and $L$ (by integration by parts), we transform this derivative into

$$
\frac{d\left\{\omega^{2} J^{\prime}(\omega)\right\}}{d \omega}=\frac{1}{\omega}\left[2 L(\omega)-1-\frac{J(\omega)}{\omega}\right] .
$$

For $\omega \geq \omega^{* *}$ the right-hand side of (A.5) is negative since $L$ is a strictly decreasing function with $L\left(\omega^{* *}\right)=0.36177$. 